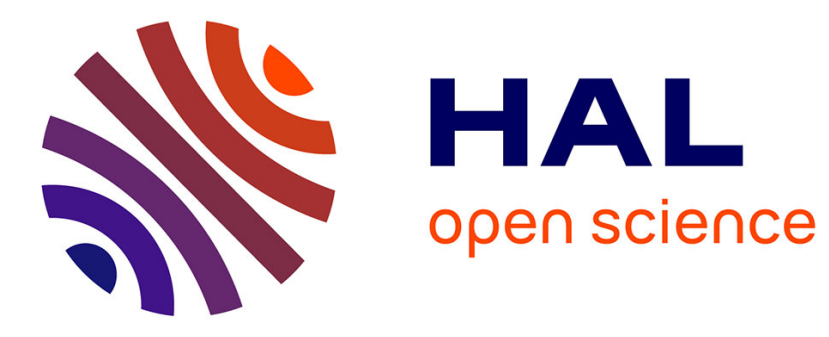

\title{
Ultra-efficient nanoparticle trapping by integrated plasmonic dimers
}

\author{
Aurore Ecarnot, Giovanni Magno, Vy Yam, Beatrice Dagens
}

\section{To cite this version:}

Aurore Ecarnot, Giovanni Magno, Vy Yam, Beatrice Dagens. Ultra-efficient nanoparticle trapping by integrated plasmonic dimers. Optics Letters, 2018, 43 (3), pp.455. 10.1364/OL.43.000455 . hal03284713

\section{HAL Id: hal-03284713 \\ https://hal.science/hal-03284713}

Submitted on 13 Jul 2021

HAL is a multi-disciplinary open access archive for the deposit and dissemination of scientific research documents, whether they are published or not. The documents may come from teaching and research institutions in France or abroad, or from public or private research centers.
L'archive ouverte pluridisciplinaire HAL, est destinée au dépôt et à la diffusion de documents scientifiques de niveau recherche, publiés ou non, émanant des établissements d'enseignement et de recherche français ou étrangers, des laboratoires publics ou privés. 


\title{
Ultra efficient nanoparticles trapping by integrated plasmonic dimers
}

\author{
AURORE ECARNOT*, GIOVANNI MAGNO, VY YAM AND BEATRICE \\ DAGENS.
}

Centre de Nanosciences et de Nanotechnologies, CNRS, Université Paris-Sud, Université Paris-Saclay, C2N-Orsay, 91405 Orsay Cedex, France
*Corresponding author: aurore.ecarnot@u-psud.fr

Received XX Month XXXX; revised XX Month, XXXX; accepted XX Month XXXX; posted XX Month XXXX (Doc. ID XXXXX); published XX Month XXXX

\begin{abstract}
We numerically demonstrate that gold dimers coupled with a SOI waveguide enable an efficient plasmonic tweezing of dielectric nanobeads, having radii down to 50 nm. By means of rigorous 3D FDTD and simplified gradient force-based calculations, we investigate the effect of the gap size involved on the tweezing action. We also demonstrate that the scattering force helps the trapping in the proximity of the dimer thanks to the establishment of light vortexes. (C) 2017 Optical Society of America
\end{abstract}

OCIS codes: (350.4855) Optical tweezers or optical manipulation; (250.5403) Plasmonics; (130.3120) Integrated optics devices.

http://dx.doi.org/10.1364/OL.99.099999

Optical forces, arising from light-matter interaction as the result of their momentum exchange, are the main characters acting in the theatre of optical trapping systems. The pioneer Arthur Ashkin in 1970 demonstrated that, thanks to the radiation pressure, it is possible to trap small particles released into a liquid when two counterpropagating laser beams were focused on these small objects [1]. In dielectric systems [2], the tweezing action undergoes diffraction limit which prevents subwavelength objects to be firmly trapped [3]. Furthermore, plasmonic structures are able to concentrate light in deep subwavelength volumes and represent an enticing alternative to overcome the limits of a dielectric system. This occurs thanks to surface plasmon polaritons (SPPs) and localized surface plasmons (LSPs) at the interface between a dielectric material and noble metal nanoparticles (MNPs) [4]. By taking advantage of plasmonic resonators inside tweezing systems, very deep and narrow potential energy wells can be tailored to achieve stable optical tweezing down to subwavelength particles.

Different plasmonic structures, such as gold disks [5], nanopillars [6] and double nanoholes [7], have been proposed to trap the smallest particles. However, in these structures, the excitation is supplied by internal total reflection resulting in a weak near field excitation. To overcome this issue, we recently proposed an integrated solution consisting in a gold MNPs chain composed by nanocylindrical particles placed on top of a silicon on insulator (SOI) waveguide. The strong coupling occuring between the SOI waveguide and the plasmonic structure [8] enables a giant excitation of the MNPs chain near field [9]. This efficient near field excitation leads to a very efficient tweezing of nearby nanobeads. In [10], we demonstrated the trapping of very low refractive index biological particles $(n=1.38)$ by means of short plasmonic chains containing up to five MNPs. In particular, by optimising the chain geometry, it is possible to continuously change the trapping position along the chain as a function of the wavelength, paving the way for the conception of novel integrated optical linear nanopositioners.

A specific topology of plasmonic resonators that revealed to be very promising for optical tweezing is the dimer. A dimer consists of two juxtaposed MNPs, of an arbitrary shape and separated by a gap $g$, such as two disks [11], bowties [12] or two squares [13]. Dimers are well known because of the important enhancement of the near field in the proximity of the gap, which increases as the latter shrinks. As we will show further in this letter, this strong near field localization is essential to achieve a stable trapping of subwavelengh sized particles.

The performances of optical tweezers depend on both the size and the depth of the optomechanical potential energy well. The latter is a function of the indivisible system constituted by the optical fields, providing with a trapping centre, and the object we want to tweeze. The stiffness $k$ models the intensity of the restoring action along a given direction, exerted by the trapping centre when the particle tries to escape from the trap. It corresponds to the local curvature when the potential energy is at its minimum: the narrower the energy well, the higher the stiffness. However, it does not suffice as an exhaustive figure of merit to quantify the performances of the tweezing system. In fact, particles dispersed into a liquid are affected by the thermal agitation of the environment which needs to be compensated. As a stability criterion, Ashkin et al [14] suggested that the Brownian dynamic is compensated when the potential energy is much deeper $(\sim$ at least 10 times) than the thermal energy $\mathrm{k}_{\mathrm{B}} \mathrm{T}$ of the system. When this condition is fulfilled, the object is steadily trapped, independently of the random kicks the object will suffer because of the thermal agitation. 
In this letter, we numerically conceive ultra-effective optical tweezers thanks to the coupling by exploiting a dimer-like plasmonic resonator integrated on top of a traditional SOI waveguide. As it will be shown, this structure takes advantage of a very high coupling and of a narrow localization of the electric field in the gap between the two gold particles constituting the dimer. In particular, we will demonstrate that, by decreasing the size of the dimer gap $g$ from 70 down to $2.5 \mathrm{~nm}$, it is possible to attain a stable trapping of a polystyrene bead having a radius $r$ from 250 down to $50 \mathrm{~nm}$.

As depicted in Fig. 1(a) the dimer is constituted of two gold ellipsoidal nanocylinders with radii $r_{x}=40 \mathrm{~nm}, r_{y}=100 \mathrm{~nm}$ and a thickness $h=30 \mathrm{~nm}$. The latter is placed on top of a monomode SOI waveguide, having a height $h_{S i}=220 \mathrm{~nm}$ and a width $w_{S i}=500 \mathrm{~nm}$. The structure is submerged in water $(n=1.33)$ where polystyrene beads $\left(n_{b}=1.59\right)$ are dispersed. Fig. $1(b)$ shows the transmittance (blue) and the reflectance (red) spectra of a mode propagating through the SOI waveguide, in the case of a dimer gap $g=20 \mathrm{~nm}$ and a bead radius $r=250 \mathrm{~nm}$. The dimer's resonance occurs near 1.52 $\mu m$, where the absorbance exceeding 55\% demonstrating the high coupling between the SOI waveguide and the plasmonic dimer, despite the compactness of the latter. The calculation of the total force $\boldsymbol{F}$ acting on the bead is performed via 3D Finite Difference Time Domain (FDTD)-based numerical simulations (Lumerical FDTD Solutions). Particularly, we calculate the total electromagnetic field for a set of discrete positions that the bead can occupy on top of the MNPs. For each of these positions, we calculate the three components of the total force arising from the interaction between the bead and the dimer near field. For sake of simplicity, we can most likely assume that the bead is located at the top surface of the dimer in $z$-direction because the $z$-component of the force $F_{z}$ is always negative (i.e attractive) along this direction. In fact, a polystyrene bead with $r=250 \mathrm{~nm}(r=50 \mathrm{~nm})$ placed on the dimer's top surface and centred with respect to the same undergoes $F_{z}=-$ $4000 \mathrm{pN} / W\left(F_{z}=-300 \mathrm{pN} / W\right)$. Furthermore, because the $y$ component of the force has to be null for symmetry reasons (as we will show the dimer behave as a symmetric dipole), we assume that the bead is located along the centre of the waveguide with respect to the $y$-direction, which constitutes an equilibrium locus (stable or unstable depending either on the bead size and on the gap size) for the $y$-component of the optomechanical force.
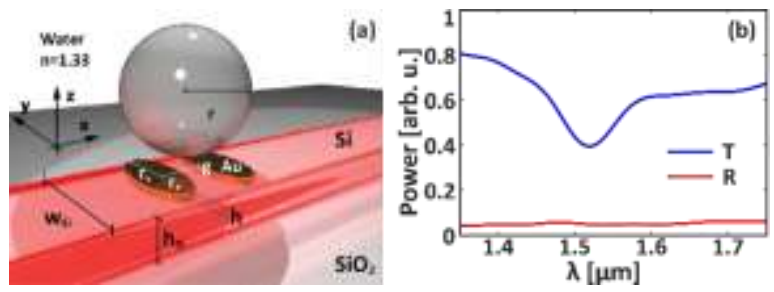

Fig. 1(a) Sketch of the proposed device. (b) Transmittance (blue) and reflectance (red) spectra for $g=20 \mathrm{~nm}$ and $r=250 \mathrm{~nm}$.

We modelled all the material involved by considering lossy and dispersive models [15]. We used ellipsometric data for the gold permittivity fitted by a Drude model with a plasma frequency $\omega_{p}=$ $1.29 \times 10^{16} \mathrm{rad} / \mathrm{s}$ and a collision frequency $\gamma=6.478 \times 10^{13} \mathrm{rad} / \mathrm{s}$. We enclosed the calculation region with perfectly matched layer (PML) boundary conditions and we used inside a variable mesh as described in [8]. In each calculation, a $10 \mathrm{~mW}$ broadband source, matching the fundamental TE mode, excites the SOI waveguide at telecom wavelengths (from 1.35 to $1.75 \mu \mathrm{m}$ ).

Fig. 2 shows the electric field squared modulus $|\mathrm{E}|^{2}$ distribution for three different decreasing gap sizes $(g=70,20$ and $2.5 \mathrm{~nm})$. For the larger gap (Fig. 2(a)), the electric field is mainly localized near each vertex of the MNPs, entailing that they are in a resonant state and behave like distinct dipoles. For gaps $\mathrm{g} \leq 20 \mathrm{~nm}$ (Fig. 2(b) and 2(c)), we observe an increased localisation and an enhancement of the electric field located between the two MNPs. More precisely, the maximum value of $|E|^{2}$ shows an enhancement of about a factor of 3 when the gap size decreases from 70 to $2.5 \mathrm{~nm}$. Because for a given excitation power deeper energy wells correspond to higher $|E|^{2}$, smaller gap sizes can be exploited to maximize the optical forces intensities and to steadily trap smaller objects.
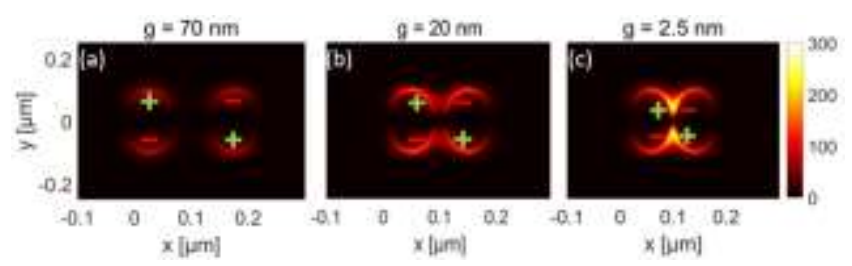

Fig. 2. Maps of $|E|^{2}$ calculated on the dimer's top facet for $g$ equal to (a) $70 \mathrm{~nm}$, (b) $20 \mathrm{~nm}$ and (c) $2.5 \mathrm{~nm}$.

As a necessary condition to trap a particle, the stiffness value needs to be negative, since this would entail the existence of a restoring action on the object. Fig. 3 shows the value of stiffness along the $x$-direction $k_{x}$, as a function of the $x$-direction and of the wavelength, calculated for a gap $g=70 \mathrm{~nm}$ (Fig. 3(a, b)) and $g=20$ $n m$ (Fig. 3(c, d)) when the bead radii $r=250 \mathrm{~nm}$ (Fig. 3(a, c)) and $r=$ $50 \mathrm{~nm}$ (Fig. 3(b, d)).

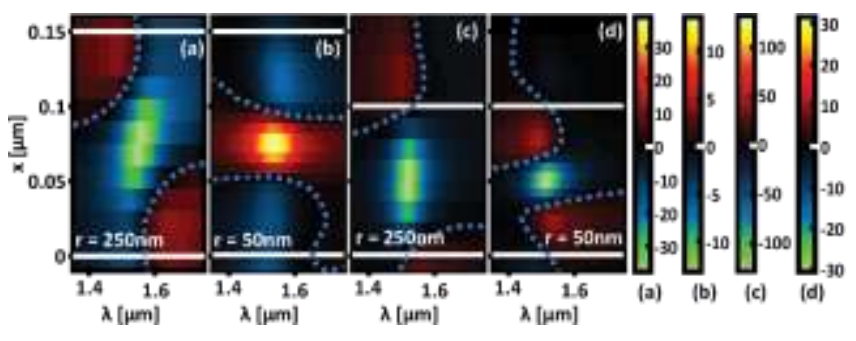

Fig. 3. Stiffness map $k_{x}[f N / n m]$ as a function of the $x$-direction and of the wavelength for $g=70 \mathrm{~nm}(\mathrm{a}, \mathrm{b})$ and $g=20 \mathrm{~nm}(\mathrm{c}, \mathrm{d})$ when $r=250 \mathrm{~nm}(\mathrm{a}$, c) and $r=50 \mathrm{~nm}(\mathrm{~b}, \mathrm{~d})$. The blue dotted curves depict the locus where the stiffness is null. The white lines indicate the middle of each MNP.

The blue dotted curves, representing the locus where the stiffness is null, delimit the states where the trapping is possible. The two white solid lines correspond to the middle of each gold particles. Fig. 3(a) shows that the maximum stiffness value occurs at resonance and when a polystyrene bead with $r=250 \mathrm{~nm}$ is located exactly in between the two MNPs, with $k_{x}=-3.77 \times 10^{3} \mathrm{fN} \cdot(\mathrm{nm} \cdot \mathrm{W})^{-1}$ (at $\lambda=1.54 \mu \mathrm{m}$ ) when a gap of $70 \mathrm{~nm}$ is considered. However, by decreasing the bead radius to $50 \mathrm{~nm}$ (Fig. 3(b)), the stiffness between the two MNPs is positive (unstable equilibrium) and the trapping is no longer possible along $y=0$ (we will show that two distinct symmetric wells form). Fig. 3(c) and (d) show that when $g$ $=20 \mathrm{~nm}$ the bead undergoes a restoring force along the $x$-direction with $k_{x}=-12.98 \times 10^{3} \mathrm{fN} \cdot(\mathrm{nm} \cdot \mathrm{W})^{-1}$ (at $\left.\lambda=1.52 \mu \mathrm{m}\right)$ and $k_{x}=-3.12 \times 10^{3}$ 
$f N \cdot(n m \cdot W)^{-1}$ (at $\lambda=1.51 \mu m$ ) when $r=250 \mathrm{~nm}$ and $r=50 \mathrm{~nm}$ respectively.

Fig. 4(a) shows the stiffness $k_{x}$ as a function of the gap size for three different values of the bead radius $(r=50,100$ and $250 \mathrm{~nm})$. We observe that the stiffness absolute value increases as the gap shrinks. In absence of a brownian dynamic, a bead with $r=250 \mathrm{~nm}$ (red curve) would be trapped regardless of the gap size within the observation range. For a bead radius $r=50 \mathrm{~nm}$ (blue curve), a negative value of the stiffness is achieved only for $\mathrm{g}<40 \mathrm{~nm}$. It is worth pointing out that, for a feasible gap size of $20 \mathrm{~nm}$ and a sized bead of $50 \mathrm{~nm}$, the stiffness absolute value is still as high as $3.12 \times 10^{3}$ $f N \cdot n m^{-1} \cdot W^{-1}$.
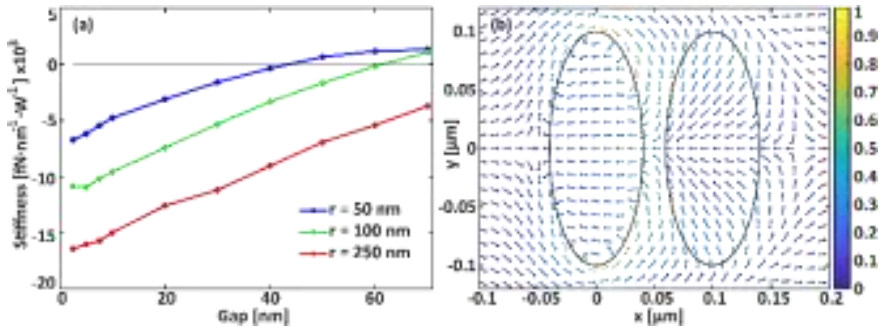

Fig. 4(a). Stiffness $k_{x}$ as a function of the gap $g$ for bead radii $r=50 \mathrm{~nm}$ (blue curve), $r=100 \mathrm{~nm}$ (green curve) and $r=250 \mathrm{~nm}$ (red curve). (b) Poynting vector calculated on the dimer's top plane as a function of the $x$ and $y$-coordinates, when $g=20 \mathrm{~nm}$ and at plasmonic resonance when $\lambda=1.53 \mu \mathrm{m}$. The intensity of the Poynting vector is given by the colorbar. The superposed black curves delimit the position of the MNPs.

However, to achieve a stable trapping in presence of thermal agitation, the Ashkin criterion needs to be satisfied. This can be accounted by calculating the potential energy distribution $U$, along a given direction, by searching for its minima and by evaluating the depth $\Delta U\left(x_{i}\right)=U(\infty)-U\left(x_{i}\right)$ for each point $x_{i}$ where a minimum occurs. In practical situations, when only the total force is available, an approximation of $U$, up to a constant term, can be obtained by integrating the total force $\boldsymbol{F}$ over the space that can be occupied by the object (in our case we consider the hemispace $z \geq r$ ). Even by limiting this calculation to only two sections for $z=r$ (along the $x$ direction when $y=0$ and along the $y$-direction when $x$ corresponds to the gap centre) with a sufficient spatial resolution, the computational efforts are massive. To circumvent this hurdle, we calculate $U$ by using the method based on the convolution between the gradient force potential density and the object (Gradient Force Density Convolution, GFDC) discussed in [10] (see Eq. (1) of this reference). Here, we calculated only the conservative force terms due to the unperturbed structure, neglecting both the perturbation due to the presence of the object on the plasmonic resonator and the contribution to non-conservative force terms arising from the field scattered by the object. This is enough to characterise the trapping operations either when the refractive index of the trapped bead is close to that of the environment or when the radiation pressure becomes negligible because of any compensation mechanism of the Poynting vector along the observation direction. And, indeed, it has been recently demonstrated in structures with an infinite chain of MNPs on top of SOI waveguide that, near the plasmonic resonance, the average value of the $x$-component of the Poynting vector is very small in the hemispace above the MNPs due to the formation of Poynting vector vortexes, associated to a slowed down light [8]. Although the dimer that we considered is composed of only two elements, a similar behaviour occurs near resonance, because of its steady-state nature. In fact, Fig. 4(b) shows the Poynting vector at the plasmonic resonance in the $[x, y]$-plane at the top facet of the dimer. For symmetry reason, along $y$-direction, the energy flow forms two symmetric vortexes near the first MNP vertices. In Fig. 4(b), in the proximity of the first MNP's vertices the Poynting vector is more intense than elsewhere (the intensity is given by the arrow colour).

Fig. 5 displays the trapping energy, normalized to the thermal energy ( $\mathrm{k}_{\mathrm{B}} \mathrm{T}$ ), obtained by means of the FDTD method (solids lines) and of the GFDC method (dashes lines) for a bead radius equals to $250 \mathrm{~nm}$ (blue and red curves) and $50 \mathrm{~nm}$ (black and green curves) as a function of $x$ (Fig. 5(a)) and $y$ (Fig.5(b)) directions.

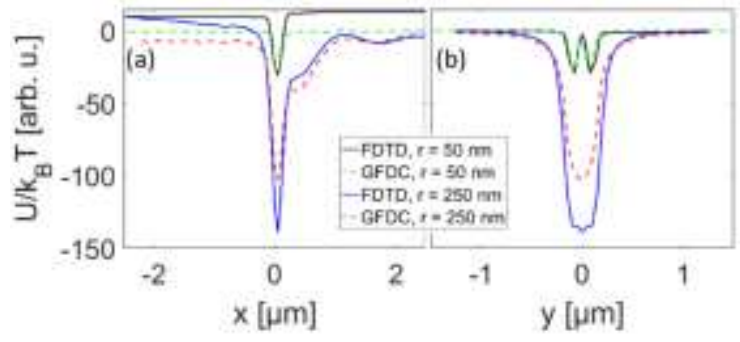

Fig. 5. Comparison between the FDTD (solid lines) and the GFDC (dashed lines) calculation of the trapping energy normalized to the thermal energy ( $\mathrm{k}_{\mathrm{B}} \mathrm{T}$ ) as a function of the (a) $x$ and (b) $y$ coordinates. These results are obtained for $g=20 \mathrm{~nm}$ and at plasmonic resonance and for $r=250 \mathrm{~nm}$ (blue and red curves) when $x=50 \mathrm{~nm}$ (a) and $y=0$ (b)) and $r=50 \mathrm{~nm}$ (black and green curves) when $x=15.9 \mathrm{~nm}$ (a) and $y$ $=76.5 \mathrm{~nm}(\mathrm{~b})$.

The reported curves are comparable in terms of shape and amplitudes. However, in Fig. 5(a) when $|x|>0.5 \mu m$, the FDTD curves appear somewhat tilted with respect to the corresponding GFDC one. Indeed, far from the MNPs and from the Poynting vector vortexes, the scattering force (taken into account only in FDTD simulation) no more cancels out over the bead volume. More precisely, the scattering term arises from the evanescent part of the waveguide propagating mode: on the left side of the dimer, the scattering force drives the particle toward the dimer, whereas on the right side it contributes to push the particle away from the dimer. Besides, far from the MNPs along the $y$-direction, the contribution of the waveguide to the scattering force is negligible. Near the dimer, the two counter-rotating vortexes formed in the MNPs vertices (as shown in Fig. 4(b)), play a positive role, enhancing the trapping along this direction. In fact, in Fig. 5(b), a difference between the two curves minimum values appears. Nevertheless, it is worth to notice that, for smaller particles, the potential well splits in two symmetric and distinct dips along the $y$ direction. Less evident differences appear between the two methods when $r=50 \mathrm{~nm}$ (black and green curves in Fig. 5), due to the less important impact of the scattering force on smaller particles. These results enable us to conclude that the GFCD method does not overestimate the optomechanical well depth and provides fast and reasonable approximation of the potential energy.

Fig. 6 shows the map of the potential energy $U$, normalized by the thermal energy, as a function of the $x$ and $y$-positions for $g=20 \mathrm{~nm}$ and $r=50 \mathrm{~nm}$, obtained by using the GFCD method. We observe the presence of two distinct potential wells (as in Fig. 5(b)) with a depth reaching the values of -26.6 when the bead is located at $x=15.9 \mathrm{~nm}$ 
and $y= \pm 76.5 \mathrm{~nm}$. This confirms that the polystyrene bead can be efficiently trapped at this position. The stiffness along the $y$ direction, estimated by the derivation of the potential energy $U$, thanks to the GFDC method, reaches a value of $-5.89 \times 10^{3} \mathrm{fN} \cdot(\mathrm{nm} \cdot \mathrm{W})$ ${ }^{-1}$ which is one order of magnitude smaller than that calculated along $x$-direction $k_{x}$ in the same case (the same behaviour have been observed experimentally in [16] for 5-elements MNP chains). Furthermore, FDTD calculations of the stiffness along the $y$ direction show a comparable value of $-4.07 \times 10^{3} \mathrm{fN} \cdot(\mathrm{nm} \cdot \mathrm{W})^{-1}$.

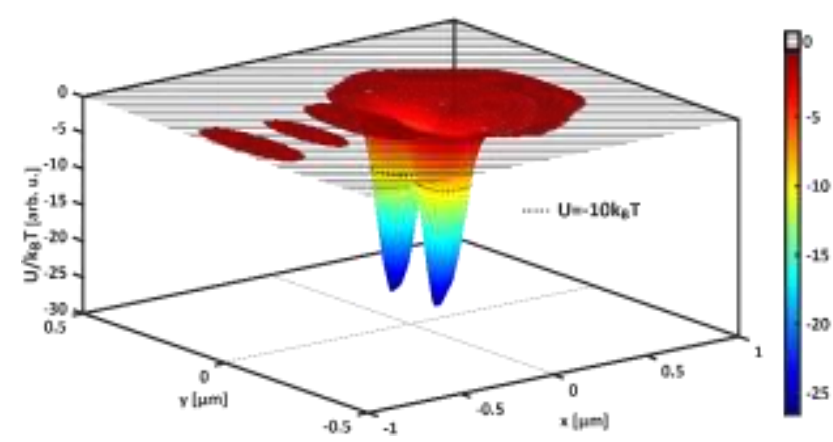

Fig. 6. Map of the normalized optomechanical energy as a function of the $x$ and $y$-directions obtained with GFCD method for $g=20 \mathrm{~nm}$ and $r=50$ $n m$. The black dashes curves correspond to the limit of the Ashkin criterion.

Fig. 7 represents the normalized potential energy minimum value (accounting for the well depth) as a function of the trapped bead radius, for a gap varying from 2.5 to $70 \mathrm{~nm}$ and calculated by using the GFDC method. On one hand, when a constant bead radius is considered, the energy well becomes deeper as the gap size shrinks. When a small radius is considered, the energy depth changes only negligibly with the gap size, entailing a large fabrication tolerance for this parameter. On the other hand, when a constant gap size is considered, the energy depth decreases as radius shrinks: the Ashkin criterion is no more satisfied when $r<25$ $n m$ regardless of the dimer gap size. In this case, the trapping can still be achieved by increasing the injected power (i.e. $30 \mathrm{~mW}$ are required to trap a bead having $r=25 \mathrm{~nm}$ ). As anticipated, for $r=50$ $\mathrm{nm}$ and $g=70 \mathrm{~nm}$ the positive stiffness at $y=0$ does not entail instability: FDTD calculation reveals that two symmetrical wells are found, with minima located at $y= \pm 76.5 \mathrm{~nm}$.

To conclude, we have numerically demonstrated the breakthrough provided by a simple structure easy to fabricate: an integrated plasmonic tweezers made of a SOI waveguide coupled with a gold dimer which enable to trap small bead with low injected power. By decreasing the gap size and thanks to light concentration in plasmonic dimers, the gradient of the electric field and then the optical forces are considerably enhanced. A high stiffness value, calculated by the FDTD method, of $-6.73 \times 10^{3} \mathrm{fN} \cdot \mathrm{nm}^{-1} \cdot \mathrm{W}^{-1}$ is obtained for a gap size of $2.5 \mathrm{~nm}$ and a bead radius of $50 \mathrm{~nm}$, which represents an improvement of a factor of 1.5 with respect to literature [13]. Furthermore, we demonstrated that, near the dimer, the occurrence of two counter-rotating Poynting vector vortexes increases the trapping stability.

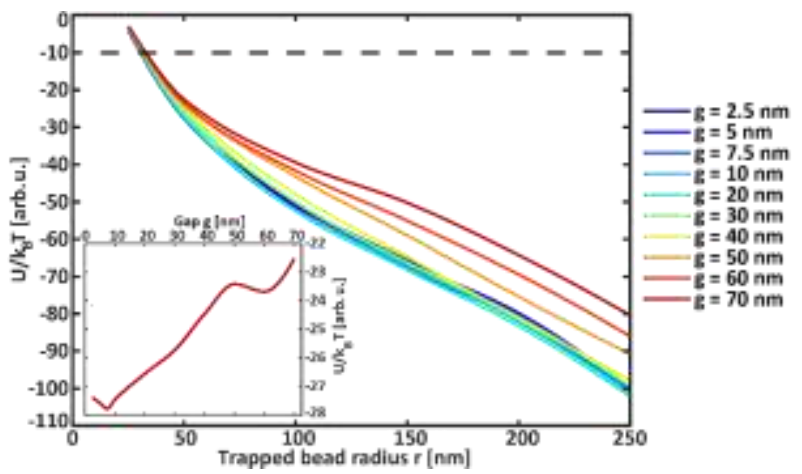

Fig. 7. Normalized trapping energy minimum as a function of the trapped bead radius for different gaps. The inset shows the same quantity as a function of the gap size when $r=50 \mathrm{~nm}$ at $y= \pm 76.5 \mathrm{~nm}$.

Finally, thanks to highly efficient dimer excitation from the SOI waveguide, the Ashkin criterion is satisfied and stable trapping is ensured for a polystyrene bead having radius down to $50 \mathrm{~nm}$ and for a power injected in the waveguide as low as $10 \mathrm{~mW}$. Additionally, a multiparameter analysis was required to identify dimer designs. We have firstly compared time-consuming complete 3D FDTD numerical simulation with a fast, semi-analytical Gradient Force Density Convolution (GFDC) method and shown the relevance and the limits of the latter for the large scan study. Main results shed light on the importance of having a very small dimer gap when dealing with the trapping of highly subwavelength beads, but also of having a large tolerance to gap size fluctuation which could occur during technological fabrication. This integrated plasmonic tweezers open the perspective to have efficient integrated sensor requiring particle immobilisation and is also suitable with low-consuming lab-on-chip design.

Funding. Paris-Sud University Chair (PSA).

\section{References}

1. A. Ashkin, Phys. Rev. Lett. 24, 156-159 (1970).

2. L. Novotny, R. Bian, and X. Xie, Phys. Rev. Lett. 79, 645-648 (1997).

3. M. L. Juan, M. Righini, and R. Quidant, Nat. Phot. 5, 349-356 (2011).

4. W. L. Barnes, A. Dereux, and T. W. Ebbesen, 424, 824-830 (2003).

5. M. Righini, G. Volpe, C. Girard, D. Petrov, and R. Quidant, Phys. Rev. Lett. 100, 8-11 (2008).

6. K. Wang and K. B. Crozier, ChemPhysChem 13, 2639-2648 (2012).

7. A. Kotnala and R. Gordon, Nano Lett. 14, 853-856 (2014).

8. G. Magno, M. Fevrier, P. Gogol, A. Aassime, A. Bondi, R. Mégy, and B. Dagens, Sci. Rep. 7, 7228 (2017).

9. M. Février, P. Gogol, A. Aassime, A. Chelnokov, A. Apuzzo, S. Blaize, J.-M. Lourtioz, and B. Dagens, Nano Lett. 12, 1032-1037 (2012).

10. G. Magno, A. Ecarnot, C. Pin, V. Yam, P. Gogol, R. Megy, B. Cluzel, and B. Dagens, Opt. Lett. 41, (2016).

11. A. N. Grigorenko, N. W. Roberts, M. R. Dickinson, and Y. Zhang, Nat. Photonics 2, 365-370 (2008).

12. B. J. Roxworthy, K. D. Ko, A. Kumar, K. H. Fung, E. K. C. Chow, G. L. Liu, N. X. Fang, and K. C. Toussaint, Nano Lett. 12, 796-801 (2012).

13. Y. Tanaka, S. Kaneda, and K. Sasaki, Nano Lett. 13, 2146-2150 (2013).

14. A. Ashkin, J. M. Dziedzic, J. E. Bjorkholm, and S. Chu, Opt. Lett. 11, 288-290 (1986).

15. E. D. Palik, Handbook of Optical Constants of Solids (1997).

16. B. Dagens, N. Yam, R. Megy, C. Pin, E. Picard, E. Hadji, and B. Cluzel, in CLEO/QELS (2015). 